\title{
Infección recurrente por Clostridium difficile en pediatría. Reporte de dos casos y revisión de la literatura
}

\section{Report of Two Cases of Recurrent Clostridium Difficile Infections in Children and Literature Review}

\author{
Wilson Daza Carreño, MD, ${ }^{1}$ Silvana Dadán, ${ }^{2}$ Michelle Higuera, MD. ${ }^{3}$
}

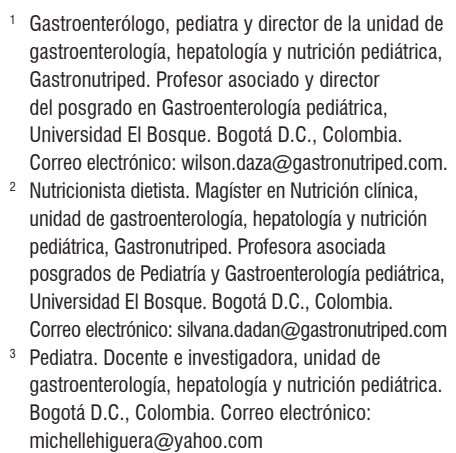

Gastroenterólogo, pediatra y director de la unidad de gastroenterología, hepatología y nutrición pediátrica, Gastronutriped. Profesor asociado y director del posgrado en Gastroenterología pediátrica, Universidad El Bosque. Bogotá D.C., Colombia. Correo electrónico: wilson.daza@gastronutriped.com.

2 Nutricionista dietista. Magíster en Nutrición clínica, unidad de gastroenterología, hepatología y nutrición pediátrica, Gastronutriped. Profesora asociada posgrados de Pediatría y Gastroenterología pediátrica, Universidad El Bosque. Bogotá D.C., Colombia. Correo electrónico: silvana.dadan@gastronutriped.com

3 Pediatra. Docente e investigadora, unidad de gastroenterología, hepatología y nutrición pediátrica. Bogotá D.C., Colombia. Correo electrónico: michellehiguera@yahoo.com

Fecha recibido: $\quad 01-05-15$ Fecha aceptado: 26-01-16

\begin{abstract}
Resumen
Antecedente: el uso indiscriminado de antibióticos no solo se relaciona con la resistencia, sino también con el aumento de algunas infecciones bacterianas, como es el caso de Clostridium difficile (CD). A pesar de un tratamiento adecuado, estas infecciones presentan una alta tasa de recurrencia, por lo que es necesario contar con pautas adecuadas de manejo y seguimiento. Metodología: se hace la presentación de 2 pacientes en edad pediátrica con episodios de reinfección por $\mathrm{CD}$, manejados en la unidad de gastroenterología, hepatología y nutrición pediátrica, Gastronutriped (Bogotá, Colombia), así como una actualización en la identificación de factores de riesgo, diagnóstico y tratamiento de la infección recurrente por $\mathrm{CD}$. Conclusiones: los casos de infección por CD presentan un reto en la población pediátrica por la similitud con cuadros infecciosos; la presencia de deposiciones sanguinolentas con el antecedente de consumo previo de antibiótico deben hacer sospechar la infección. Los casos de reinfección pueden presentarse hasta 3 meses luego del cuadro inicial; el manejo de la primera reinfección en casos leves no requiere de antibiótico, pero en casos moderados o severos se puede dar el mismo tratamiento inicial (metronidazol), en caso de una segunda reinfección se debe utilizar vancomicina, en caso de 3 o más episodios todavía hay discusiones en cuál es el tratamiento más adecuado. En los últimos años, se ha visto que el uso de probióticos y el trasplante de microbiota fecal han mostrado mayores beneficios en el cuadro de reinfección, aunque la evidencia disponible todavía es no concluyente.
\end{abstract}

Palabras clave

Clostridium difficile, pediatría, antibacterianos, diarrea.

\begin{abstract}
Background: Indiscriminate use of antibiotics is not only related to resistance, but also to increased incidence of some bacterial infections such as Clostridium difficile (CD). Despite appropriate treatment, these infections have a high recurrence rate which requires appropriate management and monitoring guidelines. Methodology: This article presents the cases of two pediatric patients who experienced CD reinfection on more than one episode. Patients were treated in Gastroenterology, Hepatology and Nutrition Unit of Gastronutriped in Bogotá, Colombia. In addition, the article provides an update on identification of risk factors, diagnosis and treatment of recurrent $C D$ infections. Conclusions: Cases of CD infections in the pediatric population present a challenge because of their similarity to other infectious processes. When there are bloody stools in a patient with a history of prior use of antibiotics, the infection should be suspected. Reinfection can occur up to 3 months after the initial infection. Management of the first reinfection in mild cases does not require antibiotics, but moderate or severe may be treated with metronidazole if it was used for initial treatment. In cases of a second reinfection, vancomycin should be used. The most appropriate treatment for cases of three or more episodes is still a matter of debate. In recent years, the use of probiotics and fecal microbiota transplantation have shown great benefits in cases of reinfection, though the available evidence is still inconclusive.
\end{abstract}

\section{Keywords}

Clostridium difficile, pediatrics, antibacterial, diarrhea. 


\section{INTRODUCCIÓN}

El Clostridium difficile (CD) es una bacteria anaerobia formadora de esporas $(1,2)$; su incidencia de infección ha ido en franco ascenso, conllevando al aumento de la mortalidad secundaria a la misma $(3,4)$. Alrededor de dos tercios de los casos ocurren en la comunidad y el tercio restante en el ámbito hospitalario (2). Si bien un alto porcentaje de niños están colonizados con CD y son portadores asintomáticos (5), son pocos los que desarrollan la enfermedad, y con adecuado manejo presentan resolución del cuadro (6, $7)$; por otro lado, aquellos casos que requieren tratamiento presentan recurrencia (RCD) hasta en un 19,6\% (8).

La microbiota intestinal desempeña múltiples funciones (7) y puede alterarse por diversos factores, entre ellos, la vía del parto (cesárea), la ausencia o corta duración de la lactancia materna, el inicio temprano de la alimentación complementaria, la prematurez $(9,10)$, el uso de agentes supresores de ácido gástrico o el uso de antimicrobianos $(7,11)$ que, individual o en conjunto, favorecen la colonización de varios patógenos, entre ellos el CD (12); en lactantes colonizados por Clostridium difficile se ha observado un aumento de Ruminococcus y de Klebsiella (13). También se ha visto infección por $\mathrm{CD}$ en pacientes con enfermedad inflamatoria intestinal (EII) (14) o con fibrosis quística (FQ) (15).

A continuación, se realiza la presentación de 2 pacientes pediátricos con infección recurrente por $\mathrm{CD}$, atendidos en la unidad de gastroenterología, hepatología y nutrición pediátrica, Gastronutriped, y se muestra una revisión complementaria de la literatura con énfasis en el manejo de la recurrencia de infección por este patógeno.

\section{PRESENTACIÓN DE CASOS CLÍNICOS}

\section{Caso clínico 1}

Se trata de un paciente de género femenino, de 3 años y 9 meses de edad, que durante el último año vive alternadamente entre Estados Unidos de América (EEUU) y Bogotá. Presenta un cuadro clínico que inició con síntomas respiratorios, por lo que asiste a servicio médico en EEUU y allí se realiza diagnóstico de bronquitis manejada con amoxicilina y ácido clavulánico por 3 días. A los 6 días de terminar el tratamiento presentó hematoquecia asociada con dolor abdominal tipo cólico de gran intensidad, por lo que consultó al servicio de urgencias en los EEUU, con toxina de CD positiva dentro de los paraclínicos solicitados; se inició tratamiento con metronidazol en $10 \mathrm{mg} / \mathrm{kg} /$ dosis, Saccharomyces boulardii por 10 días y dieta libre de lácteos.

Después de 6 días del inicio del tratamiento recurre la hematoquecia, asociada con dolor abdominal tipo cólico y esta vez con diarrea; es valorada por gastroenterología pediátrica, que reconfirmó $\mathrm{CD}$ en 3 cultivos y reinstauró metronidazol en $10 \mathrm{mg} / \mathrm{kg} /$ dosis por 21 días. La paciente presenta mejoría de los síntomas, sin embargo, a los 4 días de finalizar el tratamiento, ya en Colombia, reaparece la hematoquecia, esta vez sin dolor abdominal; consultan a infectología pediátrica y, por sospecha de recaída, se inicia vancomicina oral en dosis de $40 \mathrm{mg} / \mathrm{kg} /$ día cada 8 horas por 14 días, continúa con Saccharomyces boulardii y se adiciona sulfato de zinc.

En la primera consulta en Gastronutriped, 8 semanas después de su cuadro respiratorio inicial y de la consulta con el infectólogo pediatra, se evidencia alteración pondoestatural en el examen físico, reporta antecedentes personales y familiares de atopia; se inician estudios para descartar alergia alimentaria, enfermedad celíaca, EII o FQy se solicita colonoscopia por la recurrencia de la hemorragia digestiva baja. En su manejo, se adicionaron multivitaminas y se reajustó la dosis de vancomicina en $30 \mathrm{mg} / \mathrm{kg} /$ día, cada 6 horas por 14 días. En consultas siguientes se descartan patologías inmunitarias.

Al término del tratamiento antibiótico presentó una nueva recaída, con deposiciones tipo Bristol 6 y 7; se indicó continuar con probióticos y zinc, sin nueva terapia antibiótica. Como hallazgos de los laboratorios se encontraron toxinas A y B para CD negativas y ASCAS (inmunoglobulina G para Saccharomyces cerevisae) positivos, por lo que se indicó endoscopia digestiva alta. No fue posible la colonoscopia porque los padres no autorizaron el procedimiento. Otros estudios realizados se presentan en la tabla 1. En el último control, a las 12 semanas de la primera valoración en Gastronutriped, la paciente presentaba adecuada evolución, seguían pendientes los estudios para descartar FQ (elastasa fecal pancreática y electrolitos en sudor) y se indicó seguir con Saccharomyces boulardii, $200 \mathrm{mg}$ cada 12 horas, hasta completar 3 meses.

\section{Caso clínico 2}

Paciente de género masculino de 7 años de edad, procedente de Bogotá, con cuadro clínico de 2 meses de evolución luego del uso de antibiótico (cefuroxima $30 \mathrm{mg}$ / $\mathrm{kg} /$ día por 10 días) por rinosinusitis. El cuadro inició con deposiciones diarreicas con moco y pintas de sangre, no explosivas, por 2 días; luego pasó a deposiciones Bristol tipo 5 y 6. Consultó al servicio de urgencias de una institución local, donde iniciaron manejo ambulatorio con suero oral y probióticos (bifidobacterias y lactobacilos vivos liofilizados) sin mejoría. Fue revalorado a los 7 días, con toma de coproscópico que evidenció trofozoitos de Entamoeba histolyitica/dispar, por lo que diagnosticaron amebiasis e iniciaron tratamiento con metronidazol $(30 \mathrm{mg} / \mathrm{kg} /$ día por 7 días) y nifuroxazida ( $5 \mathrm{mg} / \mathrm{kg} /$ día por 7 días). El paciente 
Tabla 1. Resultados de pruebas de laboratorio en paciente caso clínico 1.

\begin{tabular}{|c|c|c|c|}
\hline Prueba de laboratorio & Rango normalidad* & Resultado & Interpretación \\
\hline Inmunoglobulina A & $22-159 \mathrm{mg} / \mathrm{dL}$ & $1,49 \mathrm{mg} / \mathrm{dL}$ & Disminuida \\
\hline $\begin{array}{l}\text { Inmunoglobulina E específica (por Inmunocap) para clara y yema } \\
\text { de huevo, leche, B lacto globulina y alfa lactoalbúmina, pescado, } \\
\text { camarón, soya, maní }\end{array}$ & $\begin{array}{l}\text { Negativos } \\
(<0,35 \mathrm{Ku} / \mathrm{L})\end{array}$ & Negativos & Negativos \\
\hline D-xilosa & $32-58 \mathrm{mg} / \mathrm{dL}$ & $43,4 \mathrm{mg} / \mathrm{dL}$ & Normal \\
\hline Carotenos & $40-400 \mu \mathrm{g} / \mathrm{dL}$ & $7,33 \mu \mathrm{g} / \mathrm{dL}$ & Disminuido \\
\hline $\begin{array}{l}\text { Antitransglutaminasa tisular } \\
\text { Inmunoglobulina A } \\
\text { Inmunoglobulina } G\end{array}$ & $\begin{array}{l}\text { Negativo: inferior de } 9 \mathrm{U} / \mathrm{mL} \\
\text { Negativo: inferior de } 20 \mathrm{U} / \mathrm{mL}\end{array}$ & $\begin{array}{l}\text { Negativo } \\
\text { Negativo }\end{array}$ & $\begin{array}{l}\text { Negativo } \\
\text { Negativo }\end{array}$ \\
\hline Inmunoglobulina G para Saccharomyces cerevisae & Negativo: menor de $20,1 \mathrm{U}$ & $13,28 \mathrm{KU} / \mathrm{L}$ & Positiva \\
\hline ANCAS MPO & Negativo & Negativo & Negativo \\
\hline Transferrina & $2,03-3,6 \mathrm{~g} / \mathrm{L}$ & $2,62 \mathrm{~g} / \mathrm{L}$ & Normal \\
\hline Ferritina & $7-140 \mathrm{ng} / \mathrm{mL}$ & $85,7 \mathrm{ng} / \mathrm{mL}$ & Normal \\
\hline
\end{tabular}

ANCAS MPO: anticuerpos anticitoplasma de neutrófilos.

*Fuente: Flerlage J, Engorn, B, Harriet Lane Service (Johns Hopkins Hospital). The Harriet Lane Handbook: A manual for pediatric house officers. 20th Ed. USA Elsevier Mosby, 2015.

persiste sin mejoría, por lo que fue hospitalizado e implementan ácido nalidíxico ( $50 \mathrm{mg} / \mathrm{kg}$ / día por 7 días), hidratación endovenosa y Bacillus clausii por vía oral. Después de 3 días del inicio del tratamiento aparecen membranas blanquecinas en heces. Se hicieron estudios para descartar infección por $\mathrm{CD}$, resultando positivo para toxinas $\mathrm{A}$ y $\mathrm{B}$; también se practicó una colonoscopia. Presentó un episodio febril a las 24 horas del procedimiento, por lo que prescribieron metronidazol ( $30 \mathrm{mg} / \mathrm{kg} /$ día por 7 días) y ampicilina ( $100 \mathrm{mg} / \mathrm{kg} /$ día por 7 días), completando 10 días de tratamiento. $\mathrm{Al}$ egreso hospitalario se indicó manejo con simbiótico (bifidobacterias, lactobacilos, inulina y fructooligosacáridos) y metronidazol; presentó una nueva recaída a los 5 días, por lo que se decide toma de coproscópico que no reportó microorganismos y se manejó con oxantel y pirantel ( $10 \mathrm{mg} / \mathrm{kg} /$ día por 3 días).

El paciente consultó a Gastronutriped 2 meses después del inicio de su cuadro clínico. Dos días previos a la consulta había presentado deposición Bristol 6, escasa, con tenesmo y pintas de sangre, sin presencia de otros síntomas, en manejo con Saccharomyces boulardii (250 mg cada 12 horas); se evidenció desnutrición aguda. Se consideró como diagnósticos colitis por $\mathrm{CD}$ y síndrome posenteritis debido a la diarrea persistente; se indicó gluconato de zinc (libre de azúcares), L-glutamina y Lactobacillus reuteri, suplemento proteico, y se continuó con Saccharomyces boulardii. Se empezaron estudios para EII y FQ. A los 8 días de la primera consulta en Gastronutriped reapareció la diarrea, con moco y sangre por 4 días, sin fiebre ni compromiso general; consultó al servicio de urgencias, le indicaron manejo ambulatorio con suero oral y diosmectita, continuando con Saccharomyces boulardii. En el control, a los 24 días de la primera consulta en Gastronutriped, se evidenció resolución de los síntomas y ganancia de peso; presentaba síntomas sugerentes de estreñimiento, por lo que se prescribió polietilenglicol sin electrolitos y se dieron recomendaciones nutricionales. Entre los laboratorios solicitados se observó calprotectina fecal positiva ( $>300 \mu \mathrm{g} / \mathrm{gr})$, por lo que se sospechó EII. Otros estudios realizados se presentan en la tabla 2.

En un nuevo control, alrededor de 6 meses después de la primera consulta en Gastronutriped, la calprotectina fecal persistió positiva ( $>300 \mu \mathrm{g} / \mathrm{gr})$, por lo que se decide realizar una endoscopia digestiva alta y colonoscopia (figuras 1 y 2). En las biopsias de la endoscopia digestiva alta (figura 3) se reportó: esofagitis grado I, gastritis crónica moderada con cambios reactivos foveolares, Helicobacter pylori negativo y duodenitis grado I; por lo anterior, se inició manejo con omeprazol $20 \mathrm{mg}$ cada 12 horas por 3 meses. El paciente tuvo una evolución favorable, sin sintomatología gastrointestinal y en una nueva valoración (alrededor de 7 meses y medio desde el comienzo del cuadro), la calprotectina fecal resultó negativa. Se descartaron FQy EII.

\section{DISCUSIÓN}

La infección RCD se define como un nuevo episodio de diarrea dentro de los 90 días (confirmado por estudios diagnósticos) después de la resolución del episodio inicial, durante al menos 10 días después de la interrupción/culminación de la terapia para CD (16). Hasta un $20 \%$ de los pacientes tendrá recurrencia a pesar del tratamiento con éxito de la infección 
Tabla 2. Resultados de pruebas de laboratorio en paciente caso clínico 2.

\begin{tabular}{lccc}
\hline \multicolumn{1}{c}{ Prueba de laboratorio } & Rango normalidad & Resultado & Interpretación \\
\hline Alfa 1 antitripsina fecal & Menor o igual de $54 \mathrm{mg} / \mathrm{dL}$ & $114,7 \mathrm{mg} / \mathrm{dL}$ & Aumentada \\
Calprotectina fecal & Negativa: $<30 \mu \mathrm{g} / \mathrm{gr}$ & $>300 \mu \mathrm{g} / \mathrm{gr}$ & Positiva \\
Primer control & $<30 \mu \mathrm{g} / \mathrm{gr}$ & $>300 \mu \mathrm{g} / \mathrm{gr}$ & Positiva \\
Segundo control & $<30 \mu \mathrm{g} / \mathrm{gr}$ & $<30 \mu \mathrm{g} / \mathrm{gr}$ & Negativa \\
IgG para Saccharomyces Cerevisae & Negativo: menor de $20,1 \mathrm{U}$ & $2,83 \mathrm{U}$ & Negativa \\
ANCAS MPO & Negativo & Negativo & Negativo \\
C3 & $88-155 \mathrm{mg} / \mathrm{dL}$ & $122,1 \mathrm{mg} / \mathrm{dL}$ & Normal \\
C4 & $12-32 \mathrm{mg} / \mathrm{dL}$ & $28,9 \mathrm{mg} / \mathrm{dL}$ & Normal \\
Coprocultivo & Negativo para crecimiento de & Negativo, no hay crecimiento & Negativo \\
& bacterias patógenas & de Salmonella, Shigella, Campylobacter &
\end{tabular}

ANCAS MPO: anticuerpos anticitoplasma de neutrófilos; C3: complemento fracción 3; C4: complemento fracción 4.

Fuente: Flerlage J, Engorn, B, Harriet Lane Service (Johns Hopkins Hospital). The Harriet Lane Handbook: A manual for pediatric house officers. 20th Ed. USA Elsevier Mosby, 2015.
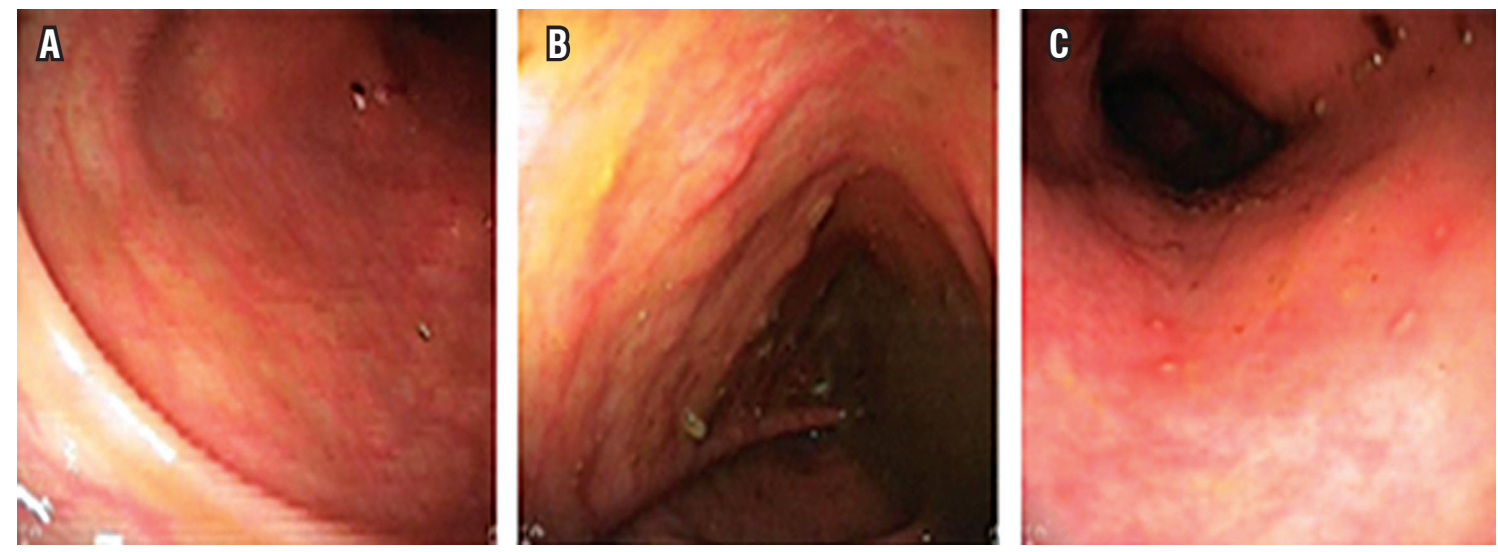

Figura 1. Imágenes de la colonoscopia. A. Mucosa eritematosa en parches sin ulceraciones, ni fístulas. B y C. Mucosa de recto sigmoide con microabscesos con halo eritematoso. Diagnóstico: proctocolitis con microabscesos.
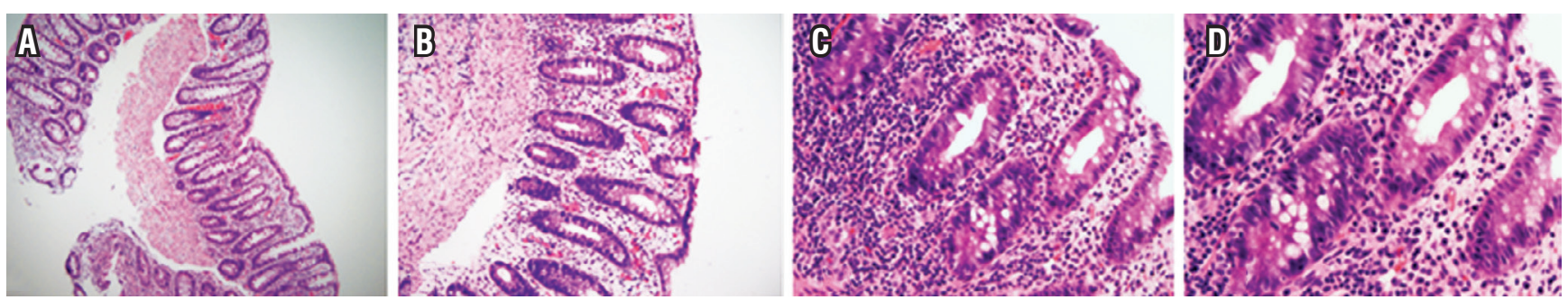

Figura 2. Informe histopatológico de las biopsias de colon. Reporte anatomopatológico. A. Colon panorámico con arquitectura preservada, criptas simétricas adosadas a muscularis mucosa. B. Acercamiento, colon con celularidad aumentada en lámina propia. C. Celularidad de mononucleares en estroma. D. Permeación de polimorfonucleares a células de las foveolas. Fuente: Cortesía Dr. Eduardo Yaspe, MD, Patólogo.

inicial, con un riesgo del $65 \%$ para aquellos que han presentado una historia previa (17). La infección recurrente sucede con mayor frecuencia dentro de 1 semana después de la interrupción del tratamiento $(18,19)$.
Los factores de riesgo predisponentes para el desarrollo, persistencia y RCD son múltiples, la exposición a la antibioticoterapia es el factor de riesgo independiente más importante para la infección, tanto en nivel comunitario 

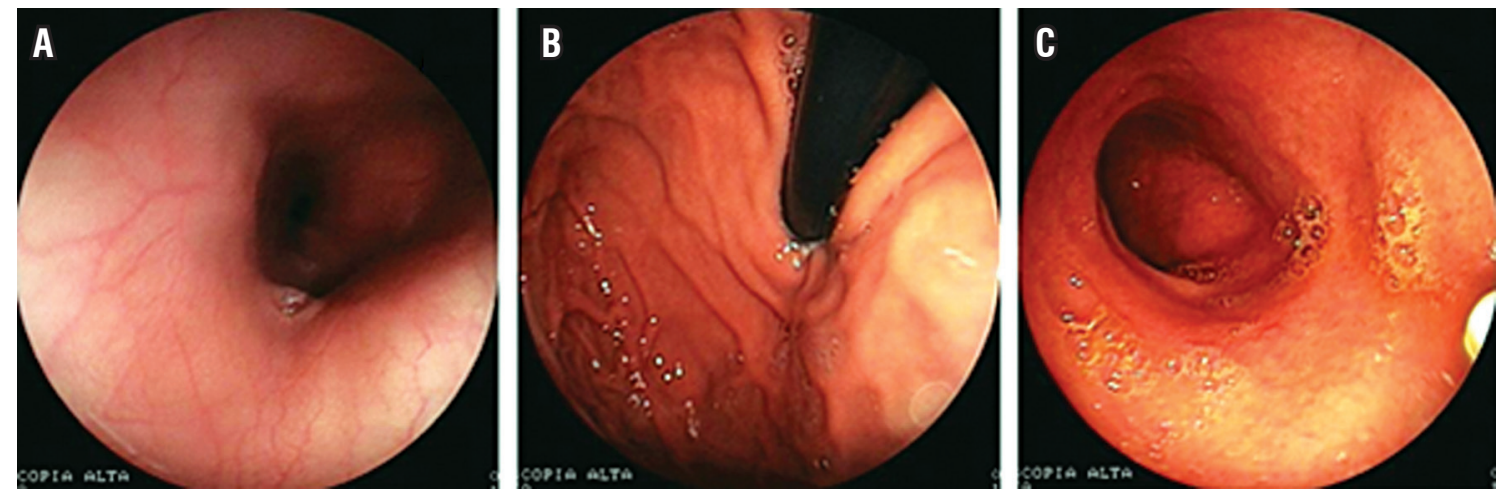

Figura 3. Imágenes de endoscopia digestiva alta. A. Esófago normal. B. Mucosa de fondo gástrico normal. C. Mucosa con eritema difusa y de aspecto nodular. Diagnóstico: duodenitis nodular.

como asociado con el cuidado de la salud (20-22), y de acuerdo con el tipo de antibiótico, el riesgo de presentación varía (tabla 3). El uso de agentes supresores de ácido gástrico es otro factor de riesgo (23, 24); sin embargo, este tópico aún está en revisión. Estudios han evaluado el efecto de estos medicamentos en niños con infección por $\mathrm{CD}$, encontrando que el 10,4\% presentaron recurrencia de la infección, siendo la recaída más frecuente cuando estaban con manejo concomitante con estos agentes (25). Otros factores de riesgo para la infección de CD que se mencionan son: hospitalización previa, procedimientos quirúrgicos previos, inmunosupresión, y uso de sondas oronasogástricas (24). Dentro de los factores asociados con RCD se mencionan: severidad de la enfermedad, edades extremas en los pacientes, y antibioticoterapia paralela por otra infección (1). Los casos expuestos en este artículo tuvieron recurrencia de la enfermedad pero no presentaron estos factores de riesgo.

Tabla 3. Clasificación de antibióticos de acuerdo con el riesgo de infección por $\mathrm{CD}$.

\begin{tabular}{|c|c|}
\hline Clasificación riesgo & Grupo de antibiótico \\
\hline Riesgo bajo & $\begin{array}{l}\text { Aminoglucósidos } \\
\text { Vancomicina } \\
\text { Trimetropin } \\
\text { Tetraciclinas } \\
\text { Penicilinas (Naturales y antipseudomonas) }\end{array}$ \\
\hline Riesgo intermedio & $\begin{array}{l}\text { Macrólidos } \\
\text { Aminopenicilinas }\end{array}$ \\
\hline Riesgo alto & $\begin{array}{l}\text { Cefalosporinas de } 2 \text { da. y 3ra. generación } \\
\text { Lincosamidas } \\
\text { Quinolonas }\end{array}$ \\
\hline
\end{tabular}

Fuente: adaptado de Stanley JD. Clostridium difficile infection. Current Problems in Surgery 2013; 50:302-337; McFee, Robin B. Clostridium difficile. Dis Mon 2009;55:439-470.
El espectro clínico de la infección por CD puede fluctuar desde una colonización asintomática, un cuadro leve, hasta una presentación severa-complicada (tabla 4). La severidad y mortalidad de la infección se asocian con las edades extremas, sin embargo, el espectro puede presentarse en cualquier tipo de edad $(1,2,26,27)$; cabe destacar que se ha reportado que los síntomas pueden ocurrir hasta 2 o 3 meses después de la exposición a antibióticos u otro factor de riesgo ( 1 , $2,18)$. En los pacientes-estudio, las manifestaciones clínicas encontradas fueron leves, los sintomas se presentaron 1 semana después del término de los antibióticos, lo que se correlaciona con los hallazgos de la literatura $(1,2,21)$.

Tabla 4. Clasificación de la severidad en niños con infección por Clostridium difficile.

\begin{tabular}{ll}
\hline \multicolumn{1}{c}{ Grado de severidad } & \multicolumn{1}{c}{ Hallazgos clínicos y/o paraclínicos } \\
\hline Leve & Afebril \\
& Diarrea (sin síntomas sistémicos) \\
Moderada & Fiebre \\
& Diarrea profusa \\
& Dolor abdominal \\
Severa & Fiebre \\
& Diarrea profusa \\
& Dolor abdominal \\
& Distensión abdominal \\
& Creatinina elevada de acuerdo con la edad \\
& Leucocitosis (>15 000) \\
& Albúmina $<2,5$ gr/dL \\
& Colitis pseudomembranosa \\
& Hipertensión \\
& Choque \\
& Íleo \\
Severa-complicada & Megacolon tóxico \\
\end{tabular}

Fuente: modificado de Crews J, Edwards M, Torchia M. Clostridium diffici$l e$ infection in children: Clinical features and diagnosis. Actualizado 2015. No existe un consenso de la definición de infección por CD en niños. Los determinantes de severidad deben ser guiados por juicio clínico. 
Egressy y colaboradores describieron adultos con infección por $\mathrm{CD}$ y FQ, encontrando síntomas atípicos y mayor riesgo de pancolitis; por tanto, debe sospecharse la infección cuando los pacientes tienen otra comorbilidad, como por ejemplo la $\mathrm{FQ}$ y si se conjugan varios factores de riesgo (28). El diagnóstico se basa en una combinación de hallazgos clínicos y de laboratorio: diarrea clínicamente significativa, una prueba positiva por CD o hallazgos de colitis pseudomembranosa por colonoscopia (24).

Los estudios diagnósticos de referencia en los últimos 30 años han sido el ensayo de neutralización de la citotoxina CD y el cultivo toxigénico en muestra de materia fecal, sin embargo este último, a pesar de su alta prescripción, no se recomienda actualmente debido a que no logra la detección de cepas productoras de toxina (29), factor importante considerando la alta frecuencia de colonización en niños pequeños (30). La prueba de inmunoensayo enzimático de heces para la toxina A y/o B es ampliamente utilizada para el diagnóstico de la infección por $\mathrm{CD}$; en los últimos años se han implementado y estudiado otras pruebas, como la detección del antígeno específico para la enzima glutamato deshidrogenasa (enzima producida por todas las cepas de $\mathrm{CD}$ ), pero presentan como limitación que no pueden distinguir entre cepas toxigénicas y no toxigénicas, solo es útil como prueba de tamizaje en un algoritmo diagnóstico, donde las muestras positivas se someten a pruebas adicionales (31), lo que podría ser considerado una alternativa futura (2). Las toxinas A y B resultaron positivas en los pacientes presentados, confirmando el diagnóstico; luego se tornaron negativas al finalizar el tratamiento, lo que ayudó en el seguimiento de estos pacientes.

Algunos estudios reportan leucocitosis o leucopenia, neutrofilia, alteración de la función renal, aumento en reactantes de fase aguda, no obstante, si estos parámetros se ubican en rangos de normalidad, tal como acontece en nuestros pacientes-estudio, no excluyen el diagnóstico y resultan más útiles para evaluar complicaciones $(1,2,29)$. Feghaly y colaboradores mostraron que el recuento de neutrófilos, la proteína $\mathrm{C}$ reactiva y las citocinas proinflamatorias estaban elevados en pacientes con enfermedad grave en comparación con controles asintomáticos (32).

La endoscopia digestiva rara vez se utiliza para diagnosticar la infección por $\mathrm{CD}$, además que puede conllevar complicaciones, sin embargo, puede estar justificada en situaciones de urgencia, cuando se sospeche infección $y$ las pruebas de heces sean negativas $(27,33)$. En niños con diarrea crónica y con alteración del estado nutricional es importante evaluar malabsorción intestinal y descartar otros diagnósticos diferenciales, tal como se hizo en los pacientes-estudio (34). Se ha evaluado la calprotectina fecal dado que es uno de los biomarcadores más estables y proporciona información sobre el estado inflamatorio del intestino, es de gran utilidad para el seguimiento y para predecir recaídas en algunas patologías de origen inflamatorio (35); inicialmente, se describió para EII y en la actualidad, comienza a implementarse en otras patologías (36). En el caso 2, la calprotectina fecal estaba aumentada, aspecto que determinó su seguimiento, en concordancia con lo anterior, este paciente presentó un resultado positivo probablemente por la colitis por el CD y no porque padeciera EII. Sin embargo, faltan estudios para determinar la sensibilidad, la especificidad y la utilidad de este estudio en pacientes con infección por CD. En la tabla 5 se presenta la comparación de principales hallazgos en los casos clínicos y lo reportado en la literatura.

Como primer paso en el tratamiento, la Academia Americana de Pediatría recomienda suspender el antibiótico que llevó al episodio (37), con este manejo, hasta en un tercio de los casos el cuadro mejorará (38); si se presenta deshidratación, apariencia tóxica y enfermedad severa, se debe iniciar la hospitalización (39). Como manejo antibiótico, en un primer episodio se sugiere iniciar metronidazol; en los casos de una primera recurrencia, los niños con síntomas leves se pueden manejar de forma conservadora sin manejo antibiótico (40), aquellos con cuadro moderado a severo suelen responder a un segundo curso del mismo tratamiento (metronidazol) o uso de vancomicina (40). En casos de una segunda (o tercera) recurrencia o cuando se decida terapia prolongada, se recomienda siempre uso de vancomicina (40); en casos de recurrencia después de este último régimen, se han descrito beneficios con el uso de trasplante de microbiota fecal (41-44), mostrando tasas de curación de $91 \%$ a $98 \%$; sin embargo, todavía se debe determinar la ruta óptima de administración, y la evidencia es insuficiente respecto de las consecuencias y/o complicaciones de este trasplante fecal A corto y largo plazo, algunos han reportado colitis inflamatoria después del trasplante (45). En los casos-estudio, los pacientes presentaron recaída luego de la implementación del tratamiento, de hecho, la paciente del caso 1 requirió vancomicina oral. En este artículo se propone un algoritmo para el diagnóstico y tratamiento de la infección por CD (figura 4).

Se han descrito otro tipo de abordajes como es el uso de inmunoglobulina, rifaximina, probióticos, o nitazoxanida, aunque faltan estudios que los soporten para poder generar una recomendación al respecto $(37,46)$; sin embargo, 2 metaanálisis recientes apoyan el uso de probióticos en el manejo de infecciones por CD. El realizado por Mcfarland encontró que, aquellos pacientes manejados con $S$. boulardii, presentaron un RR =0,50 (IC 95\%, 0,29-0,85), con L. casei DN114001, RR =0,07 (IC 95\%, 0,01-0,55), la mezcla de $L$. acidophilus y Bifido. Bifidum, RR =0,41 (IC 95\%, 0,21-0,80), y la mezcla de $L$. acidophilus, $L$. casei y $L$. rhamnosus, $R R=0,21$ (IC 95\%, 0,11-0,40) (47). En el metaanálisis de Szajewska y 
Tabla 5. Infección por Clostridium Difficile, comparación de principales hallazgos en casos clínicos y en la literatura.

\begin{tabular}{|c|c|c|}
\hline Reporte en la literatura & Caso clínico 1 & Caso clínico 2 \\
\hline \multicolumn{3}{|l|}{ Síntomas } \\
\hline $\begin{array}{l}\text { Diarrea } \\
\text { Hematoquecia } \\
\text { Dolor abdominal } \\
\text { Fiebre }\end{array}$ & $\begin{array}{l}\text { Sí } \\
\text { Sí } \\
\text { Sí } \\
\text { Sí }\end{array}$ & $\begin{array}{l}\text { Sí } \\
\text { Sí } \\
\text { Sí } \\
\text { Sí }\end{array}$ \\
\hline \multicolumn{3}{|l|}{ Estado nutricional: } \\
\hline Normal o desnutrición & Desnutrición crónica & Desnutrición aguda \\
\hline \multicolumn{3}{|l|}{ Factores de riesgo } \\
\hline $\begin{array}{l}\text { Hospitalización previa } \\
\text { Procedimientos quirúrgicos previos } \\
\text { Inmunosupresión } \\
\text { Uso de supresores de ácido gástrico } \\
\text { Uso de antibiótico }\end{array}$ & $\begin{array}{l}\text { No } \\
\text { No } \\
\text { No } \\
\text { No } \\
\text { Sí (antibióticos de } \\
\text { riesgo intermedio) }\end{array}$ & $\begin{array}{l}\text { No } \\
\text { No } \\
\text { No } \\
\text { No } \\
\text { Sí (antibióticos de riesgo alto) }\end{array}$ \\
\hline \multicolumn{3}{|l|}{ Método de detección } \\
\hline $\begin{array}{l}\text { Cultivo } \\
\text { Inmunoensayo enzimático toxina A } \\
\text { Inmunoensayo enzimático toxina B } \\
\text { Inmunoensayo enzimático toxina A y B } \\
\text { Estudio de citotoxicidad en cultivo celular } \\
\text { Reacción de la cadena de la polimerasa }\end{array}$ & $\begin{array}{l}\text { No se tomó } \\
\text { No se tomó } \\
\text { No se tomó } \\
\text { Positivo } \\
\text { No se tomó } \\
\text { No se tomó }\end{array}$ & $\begin{array}{l}\text { No se tomó } \\
\text { No se tomó } \\
\text { No se tomó } \\
\text { Positivo } \\
\text { No se tomó } \\
\text { No se tomó }\end{array}$ \\
\hline \multicolumn{3}{|l|}{ Procedimiento endoscópico } \\
\hline $\begin{array}{l}\text { Colonoscopia: mucosa hiperémica, membranas blanco } \\
\text { amarillentas, no siempre se observan las membranas }\end{array}$ & $\begin{array}{l}\text { Los padres no la } \\
\text { autorizaron }\end{array}$ & $\begin{array}{l}\text { Reporte histopatológico extrainstitucional: colitis crónica inespecífica } \\
\text { con focos de actividad }\end{array}$ \\
\hline \multicolumn{3}{|l|}{ Tratamiento } \\
\hline $\begin{array}{l}\text { Antibioticoterapia (metronidazol - 1era. Línea) } \\
\text { Probióticos (Lactobacillus GG o Saccharomyces boulardii) }\end{array}$ & $\begin{array}{l}\text { Metronidazol } \\
\text { Saccharomyces } \\
\text { boulardii }\end{array}$ & $\begin{array}{l}\text { Metronidazol } \\
\text { Saccharomyces boulardii }\end{array}$ \\
\hline RECAÍDA & Sí & Sí \\
\hline \multicolumn{3}{|l|}{ Método de detección } \\
\hline $\begin{array}{l}\text { Cultivo } \\
\text { Inmunoensayo enzimático toxina A } \\
\text { Inmunoensayo enzimático toxina B } \\
\text { Inmunoensayo enzimático toxina A y B } \\
\text { Estudio de citotoxicidad en cultivo celular } \\
\text { Reacción de la cadena de la polimerasa }\end{array}$ & $\begin{array}{l}\text { No se tomó } \\
\text { No se tomó } \\
\text { No se tomó } \\
\text { Positivo } \\
\text { No se tomó } \\
\text { No se tomó }\end{array}$ & $\begin{array}{l}\text { No se tomó } \\
\text { No se tomó } \\
\text { No se tomó } \\
\text { Positivo } \\
\text { No se tomó } \\
\text { No se tomó }\end{array}$ \\
\hline \multicolumn{3}{|l|}{ Procedimiento endoscópico posrecaída } \\
\hline Endoscopia digestiva alta: mucosa hiperémica. Gastritis & $\begin{array}{l}\text { Los padres no la } \\
\text { autorizan }\end{array}$ & $\begin{array}{l}\text { Esófago normal. Mucosa de fondo gástrico normal. Mucosa con } \\
\text { eritema difuso y de aspecto nodular. Duodenitis nodular } \\
\text { Reporte histopatológico: esofagitis grado I, gastritis crónica moderada } \\
\text { con cambios reactivos foveolares. Helicobacter P. negativo. } \\
\text { Duodenitis grado I, hallazgos que sugieren etiología péptica. }\end{array}$ \\
\hline $\begin{array}{l}\text { Colonoscopia: mucosa hiperémica, membranas blanco } \\
\text { amarillentas. No siempre se observan las membranas }\end{array}$ & $\begin{array}{l}\text { Los padres no la } \\
\text { autorizan }\end{array}$ & $\begin{array}{l}\text { Mucosa eritematosa en parches sin ulceraciones ni fistulas. Mucosa de } \\
\text { recto sigmoide con microabscesos con halo eritematoso. Proctocolitis con } \\
\text { microabscesos. } \\
\text { Reporte histopatológico: colon descendente con edema y hemorragia } \\
\text { en lámina propia, } 9 \text { eosinófilos por campo de gran aumento. Proctitis } \\
\text { histológicamente aguda leve no específica. Conteo de } 12 \text { eosinófilos por } \\
\text { campo de gran aumento. }\end{array}$ \\
\hline \multicolumn{3}{|l|}{ Tratamiento recaída } \\
\hline $\begin{array}{l}\text { Antibioticoterapia (vancomicina o metronidazol) } \\
\text { Probióticos (Lactobacillus GG o Saccharomyces boulardii) }\end{array}$ & $\begin{array}{l}\text { Vancomicina } \\
\text { Saccharomyces } \\
\text { boulardii }\end{array}$ & $\begin{array}{l}\text { Ninguno } \\
\text { Saccharomyces boulardii }\end{array}$ \\
\hline
\end{tabular}

Fuente: modificado de Stanley JD. Clostridium difficile infection. Current Problems in Surgery 2013; 50:302-337; McFee, Robin B. Clostridium difficile. Dis Mon 2009;55:439-470. 


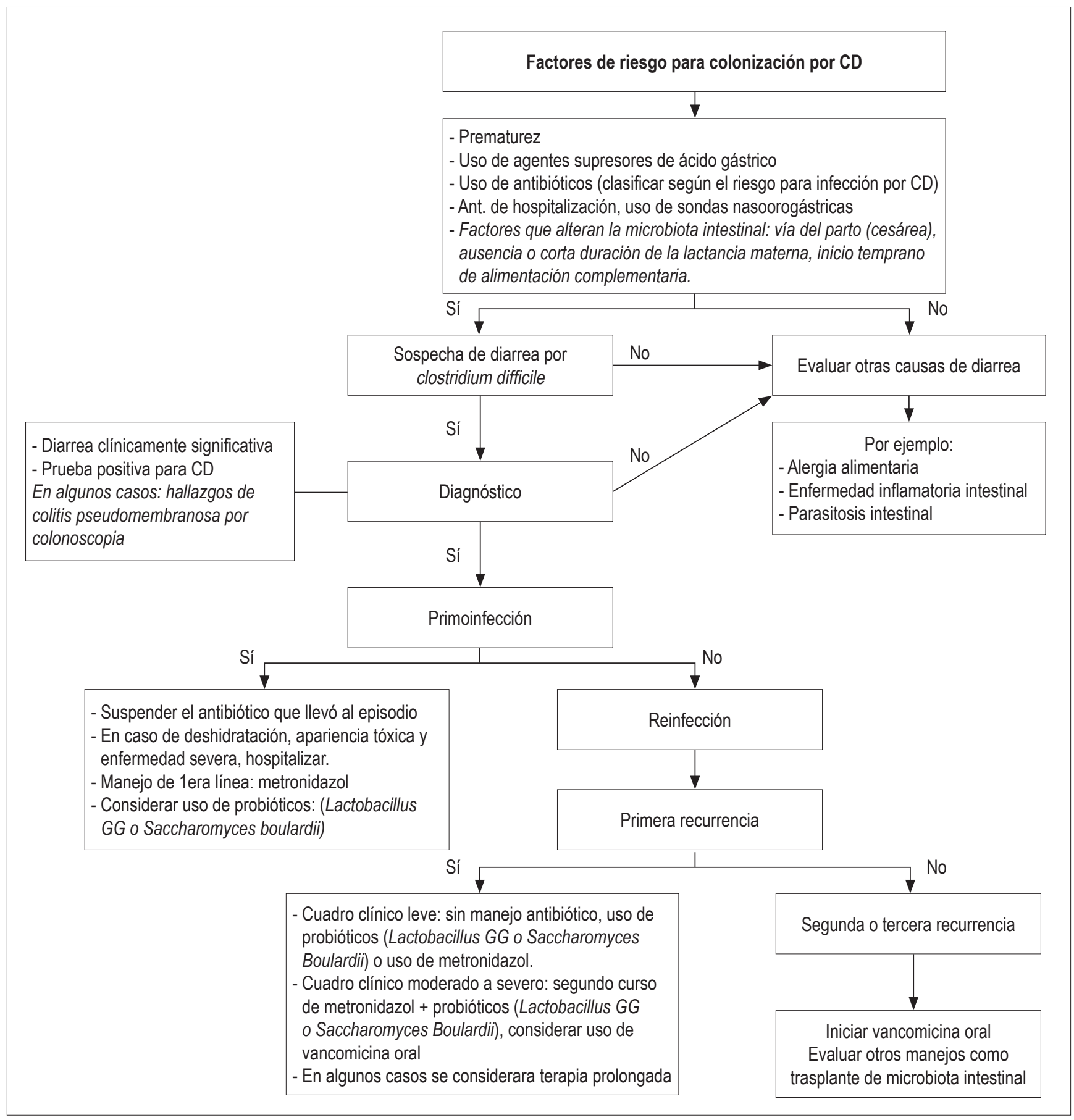

Figura 4. Propuesta de los autores para abordaje del paciente con infección por CD.

colaboradores sobre niños manejados con S. boulardii, se evidenció una diminución en el riesgo de diarrea en pacientes con $\mathrm{CD}$, con un $\mathrm{RR}=0,25$ (IC 95\%, 0,08-0,73) (48).

La inmunoglobulina intravenosa monoclonal podría ser una opción para tratar pacientes que han fracasado con la terapia inicial o para pacientes gravemente enfermos $(27$,
33), sin embargo, faltan más estudios para evaluar su uso y costo-efectividad (27). La fidaxomicina sería una alternativa para el tratamiento, con la ventaja adicional de escasos efectos negativos sobre el recuento de Bacteroides, por lo que resultaría benéfico para la microbiota intestinal (49). Asimismo, se ha evaluado una mezcla sintética de microbiota intestinal 
para tratar de reducir la transmisión de patógenos, no obstante, faltan estudios al respecto $(50,51)$. Actualmente, está en experimentación una vacuna contra el toxoide difficile que podría ser útil para prevenir la recurrencia (27).

\section{CONCLUSIONES}

Los casos de infección por $\mathrm{CD}$ en la población pediátrica presentan un reto para el personal médico por la similitud con un cuadro infeccioso y el alto riesgo de reinfección; la presencia de deposiciones sanguinolentas con el antecedente de consumo previo de antibiótico debe hacer sospechar la infección. El tratamiento de la primera reinfección, en casos leves, no requiere de antibiótico y muchos mejorarán con la suspensión del tratamiento antibiótico; en casos moderados o severos se puede dar el tratamiento inicial o uso de vancomicina. En nueva recurrencia, se recomienda el uso de vancomicina; en los casos de 3 o más episodios, todavía no existe un consenso en cuál es el tratamiento más adecuado. En los últimos años, se ha visto que el uso de probióticos ha mostrado mayores beneficios en el cuadro de reinfección, aunque la evidencia disponible es contradictoria.

Es de suma importancia evitar el uso indiscriminado de antibióticos, racionalizando las indicaciones en pediatría, en especial en las infecciones respiratorias y gastrointestinales por su alta etiología viral. Consideramos que la implementación de probióticos podría tener implicaciones favorables en el manejo de pacientes con infección por CD recurrente, con mayor beneficio en los casos leves que no requieren manejo con antibióticos, y como complemento en los casos moderados o severos.

\section{Fuentes de financiación}

Este trabajo se desarrolló con el apoyo financiero de la unidad de gastroenterología, hepatología y nutrición pediátrica (Gastronutriped).

\section{Conflicto de intereses}

Los autores declaran no tener ningún conflicto de intereses.

\section{REFERENCIAS}

1. Stanley JD, Bartlett JG, Dart BWt, Ashcraft JH. Clostridium difficile infection. Curr Probl Surg. 2013 Jul;50(7):302-337.

2. McFee RB, Abdelsayed GG. Clostridium difficile. Dis Mon. 2009;55(7):439-470.

3. Loo VG, Poirier L, Miller MA, Oughton M, Libman MD, Michaud S, et al. A predominantly clonal multi-institutional outbreak of Clostridium difficile-associated diarr- hea with high morbidity and mortality. N Engl J Med. 2005;353(23):2442-2449.

4. Borali E, Ortisi G, Moretti C, Stacul EF, Lipreri R, Gesu $\mathrm{GP}$, et al. Community-acquired Clostridium difficile infection in children: A retrospective study. Dig Liver Dis. 2015;47(10):842-846.

5. Collignon A, Ticchi L, Depitre C, Gaudelus J, Delmee M, Corthier G. Heterogeneity of Clostridium difficile isolates from infants. Eur J Pediatr. 1993;152(4):319-322.

6. Rousseau C, Poilane I, De Pontual L, Maherault A-C, Le Monnier A, Collignon A. Clostridium difficile carriage in healthy infants in the community: A potential reservoir for pathogenic strains. Clin Infect Dis. 2012;55(9):1209-1215.

7. Seekatz AM, Young VB. Clostridium difficile and the microbiota. J Clin Invest. 2014;124(10):4182-819.

8. Khanna S, Baddour LM, Huskins WC, Kammer PP, Faubion WA, Zinsmeister AR, et al. The epidemiology of Clostridium difficile infection in children: A population-based study. Clin Infect Dis. 2013;56(10):1401-146.

9. Claesson MJ, Cusack S, O’Sullivan O, Greene-Diniz R, de Weerd H, Flannery E, et al. Composition, variability, and temporal stability of the intestinal microbiota of the elderly. Proc Natl Acad Sci USA. 2011;108(1):4586-4591.

10. Hopkins MJ, Sharp R, Macfarlane GT. Age and disease related changes in intestinal bacterial populations assessed by cell culture, 16S rRNA abundance, and community cellular fatty acid profiles. Gut. 2001;48(2):198-205.

11. Zanella Terrier MC, Simonet ML, Bichard P, Frossard JL Recurrent Clostridium difficile infections: The importance of the intestinal microbiota. World J Gastroenterol. 2014;20(23):7416-7423.

12. Faber F, Bäumler AJ. The impact of intestinal inflammation on the nutritional environment of the gut microbiota. Immunol Lett. 2014;162(2):48-53.

13. Rousseau C, Levenez F, Fouqueray C, Doré J, Collignon A, Lepage P. Clostridium difficile colonization in early infancy is accompanied by changes in intestinal microbiota composition. J Clin Microbiol. 2011;49(3):858-865.

14. Nagalingam NA, Lynch SV. Role of the microbiota in inflammatory bowel diseases. Inflamm Bowel Dis. 2012 May;18(5):968-984.

15. Com G, Cetin N, O’Brien CE. Complicated Clostridium diffcile colitis in children with cystic fibrosis: Association with gastric acid suppression? J Cyst Fibros. 2014;13(1):37-42.

16. Abdelfatah M, Nayfe R, Nijim A, Enriquez K, Ali E, Watkins $\mathrm{RR}$, et al. Factors Predicting Recurrence of Clostridium diffcile Infection (CDI) in Hospitalized Patients: Retrospective Study of More Than 2000 Patients. J Investig Med. 2015;63(5):747-751.

17. Hu MY, Katchar K, Kyne L, Maroo S, Tummala S, Dreisbach $\mathrm{V}$, et al. Prospective derivation and validation of a clinical prediction rule for recurrent Clostridium difficile infection. Gastroenterology. 2009;136(4):1206-1214.

18. Olson MM, Shanholtzer CJ, Lee JT, Gerding DN. Ten years of prospective Clostridium difficile-associated disease 
surveillance and treatment at the Minneapolis VA Medical Center, 1982-1991. Infect Control Hosp Epidemiol. 1994;15(6):371-381.

19. Bartlett JG. Narrative review: the new epidemic of Clostridium difficile-associated enteric disease. Ann Intern Med. 2006;45(10):758-764.

20. Deshpande A, Pasupuleti V, Thota P, Pant C, Rolston DDK, Sferra TJ, et al. Community-associated Clostridium difficile infection and antibiotics: a meta-analysis. J Antimicrob Chemother. 2013;68(9):1951-1961.

21. Sandora TJ, Fung M, Flaherty K, Helsing L, Scanlon P, Potter-Bynoe G, et al. Epidemiology and risk factors for Clostridium difficile infection in children. Pediatr Infect Dis J. 2011;30(7):580-584.

22. Tamma PD, Sandora TJ. Clostridium difficile infection in children: Current state and unanswered questions. J Pediatric Infect Dis Soc. 2012;1(3):230-243.

23. Leonard Ad, Ho KM, Flexman J. Proton pump inhibitors and diarrhea related to Clostridium difficile infection in hospitalised patients: A case-control study. Intern Med J. 2012;42(5):591-594.

24. Tschudin-Sutter S, Tamma P, Milstone AM, Perl TM. Predictors of first recurrence of Clostridium difficile infections in children. Pediatr Infect Dis J. 2014;33(4):414-216.

25. Nylund CM, Eide M, Gorman GH. Association of Clostridium difficile infections with acid suppression medications in children. J Pediatr. 2014;165:979-984.

26. Lessa FC, Mu Y, Bamberg WM, Beldavs ZG, Dumyati GK, Dunn JR, et al. Burden of Clostridium difficile infection in the United States. N Engl J Med. 2015;372(9):825-834.

27. Vecchio AL, Zacur GM. Clostridium difficile infection: An update on epidemiology, risk factors, and therapeutic options. Curr Opin Gastroenterol. 2012;28(1):1-9.

28. Egressy K, Jansen M, Meyer KC. Recurrent Clostridium difficile colitis in cystic fibrosis: An emerging problem. J Cyst Fibros. 2013; 12:92-96.

29. Shim JO. Clostridium difficile in Children: To Treat or Not to Treat? Pediatr Gastroenterol Hepatol Nutr. 2014;17(2):80-4.

30. Wendt JM, Cohen JA, Mu Y, Dumyati GK, Dunn JR, Holzbauer SM, et al. Clostridium difficile infection among children across diverse US geographic locations. Pediatrics. 2014;133(4):651-8.

31. Bagdasarian N, Rao K, Malani PN. Diagnosis and treatment of Clostridium difficile in adults: A systematic review. JAMA. 2015;313(4):398-408.

32. Feghaly RE, Stauber JL, Tarr PI, Haslam DB. Intestinal Inflammatory biomarkers and outcome in Pediatric Clostridium difficile Infections. J Pediatr. 2013;163:1697-704.

33. Aslam S, Musher DM. An update on diagnosis, treatment, and prevention of Clostridium difficile-associated disease. Gastroenterol Clin North Am. 2006;35(2):315-35.

34. Daza W, Dadán S. Síndrome de malabsorción. En: Leal Q, Francisco. El pediatra eficiente. 7a. Ed. Bogotá, Editorial Médica Panamericana; 2013: 418-428.
35. Pang T, Leach ST, Katz T, Day AS, Ooi CY. Fecal biomarkers of intestinal health and disease in children. Front Pediatr. 2014;2(6):1-12.

36. Walkiewicz D, Werlin SL, Fish D, Scanlon M, Hanaway P, Kugathasan S. Fecal calprotectin is useful in predicting disease relapse in pediatric inflammatory bowel disease. Inflamm Bowel Dis. 2008;14(5):669-673.

37. Schutze GE, Willoughby RE, Brady MT, Byington CL, Davies HD, Edwards KM, et al. Clostridium difficile infection in infants and children. Pediatrics. 2013;131(1):196-200.

38. Morinville V, McDonald J. Clostridium difficile-associated diarrhea in 200 Canadian children. Can J Gastroenterol. 2005;19(8):497-501.

39. Lübbert C, John E, von Müller L. Clostridium Difficile Infection: Guideline-Based Diagnosis and Treatment. Dtsch Arztebl Int. 2014;111(43):723-731.

40. Cohen SH, Gerding DN, Johnson S, Kelly CP, Loo VG, McDonald LC, et al. Clinical practice guidelines for Clostridium difficile infection in adults: 2010 update by the Society for Healthcare Epidemiology of America (SHEA) and the Infectious Diseases Society of America (IDSA). Infect Control Hosp Epidemiol. 2010;31(5):431-455.

41. Varier RU, Biltaji E, Smith KJ, Roberts MS, Kyle Jensen M, LaFleur J, et al. Cost-Effectiveness Analysis of Fecal Microbiota Transplantation for Recurrent Clostridium difficile Infection. Infect Control Hosp Epidemiol. 2015;36(4):438-44.

42. Surawicz CM, Brandt LJ, Binion DG, Ananthakrishnan AN, Curry SR, Gilligan PH, et al. Guidelines for diagnosis, treatment, and prevention of Clostridium difficile infections. Am J Gastroenterol. 2013;108(4):478-498.

43. Gough E, Shaikh H, Manges AR. Systematic review of intestinal microbiota transplantation (fecal bacteriotherapy) for recurrent Clostridium difficile infection. Clin Infect Dis. 2011;53(10):994-1002.

44. Walia R, Garg S, Song Y, Girotra M, Cuffari C, Fricke WF, et al. Efficacy of fecal microbiota transplantation in 2 children with recurrent Clostridium difficile infection and its impact on their growth and gut microbiome. J Pediatr Gastroenterol Nutr. 2014;59(5):565-570.

45. Vandenplas Y, Veereman G, van der Werff Ten Bosch J, Goossens A, Pierard D, et al. Fecal microbial transplantation in a one-year-old girl with early onset colitis caution advised. J Pediatr Gastroenterol Nutr. 2015;61(3):12-14.

46. Pillai A1, Nelson R. Probiotics for treatment of Clostridium difficile-associated colitis in adults. Cochrane Database Syst Rev. 2008 Jan 23;(1):CD004611.

47. McFarland LV. Probiotics for the Primary and Secondary Prevention of $C$. difficile Infections: A Meta-analysis and Systematic Review. Antibiotics. 2015;4(2):160-78.

48. Szajewska H, Kołodziej M. Systematic review with metaanalysis: Saccharomyces boulardii in the prevention of antibiotic-associated diarrhea. Aliment Pharmacol Ther. 2015;42(7):793-801. 
49. Louie TJ, Emery J, Krulicki W, Byrne B, Mah M. OPT-80 eliminates Clostridium difficile and is sparing of bacteroides species during treatment of $C$. difficile infection. Antimicrob Agents Chemother. 2009;53(1):261-263.

50. Lawley TD, Clare S, Walker AW, Stares MD, Connor TR, Raisen C, et al. Targeted restoration of the intestinal microbiota with a simple, defined bacteriotherapy resolves relapsing Clostridium difficile disease in mice. PLoS Pathog. 2012;8(10):e1002995.

51. Crews J, Edwards M, Torchia M. Clostridium difficile infection in children: Clinical features and diagnosis. Uptodate 2015. Disponible en: http://www.uptodate.com/contents/ clostridium-difficile-infection-in-children-clinical-featuresand-diagnosis. [Consultado Diciembre 08 de 2015] 